\title{
The Physical Properties of the Groups of Galaxies
}

\author{
Lorenzo Lovisari ${ }^{1,2, *(1)}$ and Stefano Ettori 1,3 (D) \\ 1 INAF-Osservatorio di Astrofisica e Scienza dello Spazio di Bologna, via Piero Gobetti 93/3, \\ 40129 Bologna, Italy; stefano.ettori@inaf.it \\ 2 Center for Astrophysics | Harvard \& Smithsonian, 60 Garden Street, Cambridge, MA 02138, USA \\ 3 INFN, Sezione di Bologna, viale Berti Pichat 6/2, 40127 Bologna, Italy \\ * Correspondence: lorenzo.lovisari@inaf.it
}

Citation: Lovisari, L.; Ettori, S. The Physical Properties of the Groups of Galaxies. Universe 2021, 7, 254.

https://doi.org/10.3390/

universe7080254

Received: 14 July 2021

Accepted: 19 July 2021

Published: 21 July 2021

Publisher's Note: MDPI stays neutral with regard to jurisdictional claims in published maps and institutional affiliations.

Copyright: (C) 2021 by the authors. Licensee MDPI, Basel, Switzerland. This article is an open access article distributed under the terms and conditions of the Creative Commons Attribution (CC BY) license (https:// creativecommons.org/licenses/by/ $4.0 /)$.
Galaxy groups consist of a few tens of galaxies bound in a common gravitational potential. They dominate the number count of the halo mass function and contain a significant fraction of the overall universal baryon budget. Being less massive than clusters, the energy that is supplied by supernovae and active galactic nuclei (AGNs) to the hot intragroup medium (IGrM) can easily exceed their gravitational binding energy. Thus, it is expected that these non-gravitational mechanisms have a strong effect on the distribution of of the baryons, making galaxy groups ideal targets to constrain the mechanisms governing the cooling-heating balance. The net effect of the various feedback processes in action in the gravitational potential wells is to change the radial distribution of the energy and mass in groups, affecting the correlations between their observed properties. Therefore, they are key to our understanding of how the bulk of matter in the Universe accretes and forms hierarchical structures and how different sources of feedback affect their gravitational collapse.

Despite their crucial role in cosmic structure formation and evolution, galaxy groups have received less attention compared to massive clusters. This is in part due to the challenges (e.g., faint $\mathrm{X}$-ray emission, low number of galaxies in optical, low $\mathrm{S} / \mathrm{N}$ in $\mathrm{SZ}$ ) associated with their detection, observation, and characterization. With the advent of eROSITA (launched in 2019), many thousands of galaxy groups will be detected by X-ray, complementing on-going and future optical (DES, Euclid, Vera Rubin), SZ (SPT-3G, ACT), and radio (LOFAR, MeerKAT) surveys, paving the way towards the exploitation of the next generation of X-ray instruments (onboard XRISM — expected to fly by 2023 - and the ESA L2 mission Athena-expected to fly in the 2030s).

To foster progress in the field of the physical properties of galaxy groups, facilitating effective cross-communication among observers, theorists, and simulators, we organized a Special Issue (https:/ / www.mdpi.com/journal/universe/special_issues/PPGG (accessed on 21 July 2021)) dedicated to the physical properties of galaxy groups. We aimed to collect and organize the latest developments in our understanding of these systems and present future prospects from both observational and theoretical points of view. This Special Issue includes five manuscripts, which we summarize briefly in the following.

\section{- "Properties of Fossil Groups of Galaxies", by Aguerri and Zarattini [1]}

Fossil groups are an ostensibly special class of galaxy systems. They are objects dominated by a single, bright, elliptical galaxy and are thought to be the latest stage in the evolution of galaxy groups. Their properties differ from the one of other galaxy groups, and since it is likely that they did not experience recent major mergers, they should represent archetypal old undisturbed systems, and are therefore important systems to study. In the review, we show the main observational and theoretical works demonstrating that these systems fall very well in the current theory of structure formation in the Universe.

- "Scaling Properties of Galaxy Groups", by Lovisari et al. [2]

The scaling relations are the result of the different physical processes at work in the intracluster medium and provide an important tool to study its thermodynamic history. 
In fact, the various processes that govern the formation of galaxy groups are suspected of systematically increasing the intrinsic scatter of the groups relations and changing their integrated properties. We overview the most recent studies on the X-ray scaling relations, obtained at the galaxy group scale, and their relations with optical properties and the supermassive Black Hole (SMBH) mass.

\section{- "Feedback from Active Galactic Nuclei in Galaxy Groups", by Eckert et al. [3]}

The formation and evolution of the physical properties in groups are a direct consequence of the interplay between galaxy evolution, the development of the intragroup medium, and feedback. Many authors have argued that feedback from SMBHs plays a crucial role in regulating the star formation rates of massive galaxies and suppressing the onset of catastrophic cooling by carving cavities and driving shocks across the medium. We review the current observational evidence for AGN feedback in nearby galaxy groups with observations at $\mathrm{X}$-ray, radio, and millimeter wavelengths and describe the theoretical advances made in recent years to interpret the heating-cooling cycle.

- "Simulating Groups and the IntraGroup Medium: The Surprisingly Complex and Rich Middle Ground between Clusters and Galaxies", by Oppenheimer et al. [4]

The influence of the feedback processes is complex and difficult to model and to reproduce in simulations. However, cosmological simulations have enabled breakthroughs in our understanding of the gas and stellar contents of groups and of the impact of groups for cosmological parameter estimation. The review focuses on how groups process their baryons in a cosmological context, discussing the current limitations and the perspectives for improving the theoretical modeling in the near future.

- "The Metal Content of the Hot Atmospheres of Galaxy Groups", by Gastaldello et al. [5]

Metals play a central role in the thermodynamic balance of galaxy systems by sustaining the cooling of their environment by means of spectral line emissions. DUe to the shallower gravitational potential of groups, feedback effects leave important marks on their gas and metal contents. Therefore, the shape of the abundance profiles can be used to investigate the impact of the feedback in the IGrM. We review the status of the metal abundance measurements in the IGrM and the progress made by simulations to reproduce and interpret those measurements.

Funding: L.L. and S.E. acknowledge financial contribution from the contracts ASI-INAF Athena 2015046-R.0, ASI-INAF Athena 2019-27-HH.0, “Attività di Studio per la comunità scientifica di Astrofisica delle Alte Energie e Fisica Astroparticellare" (Accordo Attuativo ASI-INAF n. 2017-14-H.0), and from INAF "Call per interventi aggiuntivi a sostegno della ricerca di main stream di INAF".

Conflicts of Interest: The authors declare no conflict of interest.

\section{References}

1. Aguerri, J.A.L.; Zarattini, S. Properties of Fossil Groups of Galaxies. Universe 2021, 7, 132. [CrossRef]

2. Lovisari, L.; Ettori, S.; Gaspari, M.; Giles, P.A. Scaling Properties of Galaxy Groups. Universe 2021, 7, 139. [CrossRef]

3. Eckert, D.; Gaspari, M.; Gastaldello, F.; Le Brun, A.; O'Sullivan, E. Feedback from Active Galactic Nuclei in Galaxy Groups. Universe 2021, 7, 142. [CrossRef]

4. Oppenheimer, B.D.; Babul, A.; Bahé, Y.; Butsky, I.S.; McCarthy, I.G. Simulating Groups and the IntraGroup Medium: The Surprisingly Complex and Rich Middle Ground between Clusters and Galaxies. Universe 2021, 7, 209. [CrossRef]

5. Gastaldello, F.; Simionescu, A.; Mernier, F.; Biffi, V.; Gaspari, M.; Sato, K.; Matsushita, K. The Metal Content of the Hot Atmospheres of Galaxy Groups. Universe 2021, 7, 208. [CrossRef] 